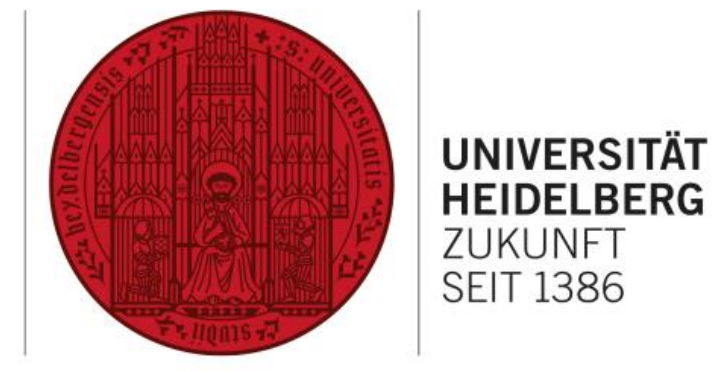

Zusammenfassung der Dissertation mit dem Titel

\title{
„Der Debt-Equity-Swap als Instrument der Unternehmenssanierung nach deutschem und englischem Recht"
}

Dissertation vorgelegt von Jonas Schwarz

Erstgutachter: Prof. Dr. Burkard Hess

Zweitgutachter: Prof. Dr. Andreas Piekenbrock

Juristische Fakultät 
Frankfurt am Main, den 8. November 2014

\section{A. Gegenstand der Untersuchung}

Gegenstand der Untersuchung ist die vergleichende Gegenüberstellung der rechtlichen Rahmenbedingungen nach deutschem und englischem Kapitalgesellschafts- und Insolvenzrecht in Bezug auf die Behandlung ausgewählter rechtlicher Problemkreise, die in der Praxis bei der Umsetzung eines Debt-Equity-Swap als Maßnahme zur finanziellen Restrukturierung von krisenbefangenen Kapitalgesellschaften regelmäßig auftreten. Hierbei wurde das Gesetz zur weiteren Erleichterung der Sanierung von Unternehmen (ESUG) berücksichtigt, das eine ganz wesentliche Zäsur für einige der untersuchten Rechtsfragen im Zusammenhang mit der Umsetzung von Debt-Equity-Swaps nach deutschem Recht darstellt.

Die Untersuchungsergebnisse sind insbesondere in den folgenden Zusammenhängen nutzbar:

1) Die rechtswissenschaftliche Diskussion der Frage, inwieweit die Attraktivität einer Umsetzung von Debt-Equity-Swaps auf der Grundlage englischen Rechts Wegzugsbemühungen deutscher sanierungsbedürftiger Unternehmen fördert und sich daher als Gefahr für den Insolvenz- und Sanierungsstandort Deutschland erweisen könnte, ist meist diffus geblieben. Sie litt darunter, dass oft nicht oder zu oberflächlich auf die rechtlichen Rahmenbedingungen von Debt-Equity-Swaps auf der Grundlage englischen Insolvenz- und Gesellschaftsrechts eingegangen wurde. Vor diesem Hintergrund hat die Untersuchung zum Ziel, die Diskussion um die tatsächlichen oder vermeintlichen Wettbewerbsnachteile des deutschen Rechts im Vergleich zum englischen Recht mit Blick auf den Aspekt der rechtlichen Rahmenbedingungen von Debt-Equity-Swaps zu bereichern und zu präzisieren, indem auch die rechtlichen Rahmenbedingungen von Debt-Equity-Swaps auf der Grundlage englischen Insolvenz- und Gesellschaftsrechts (zu ausgewählten Problemkreisen) untersucht und dargestellt werden.

2) Die Untersuchungsergebnisse liefern Antworten auf die Frage, ob bzw. inwieweit es gelungen ist, durch das ESUG der Zielsetzung des Gesetzgebers entsprechend die als Wettbewerbsnachteile geltenden Defizite des deutschen Insolvenzrechts im Vergleich zum englischen Recht abzumildern, indem die rechtlichen Rahmenbedingungen zur Umsetzung von Debt-Equity-Swaps auf der Grundlage deutschen Rechts verbessert wurden.

3) In der insolvenz- und gesellschaftsrechtlichen Beratungs- und Gestaltungspraxis kann die Erwägung eine Rolle spielen, ob es für eine deutsche Kapitalgesellschaft vorteilhaft sein könnte, eine Migration in das englische Kapitalgesellschaft- und Insolvenzrecht durchzuführen, mit dem Ziel einen Debt-Equity-Swap auf der Grundlage englischen Rechts umzusetzen. In der Wirtschaftspresse und der Fachliteratur wurde - vor Inkrafttreten des ESUG - gelegentlich der Eindruck vermittelt, dass eine solche Migration nach England zur Umsetzung eines Restrukturierungskonzepts auf der Grundlage eines englischrechtlichen Verfahrens der „Königsweg“ innerhalb der innovativen Techniken zur finanzwirtschaftlichen Sanierung deutscher Unternehmen sei. Unabhängig davon, ob das Ergebnis der Migrationserwägungen im Ergebnis für oder gegen eine Migration ausfällt, wird dann, wenn die Umsetzung eines Debt-Equity-Swap als finanzielle Sanierungsmaßnahme erwogen wird (neben einer Vielzahl anderer in die Abwägung einzustellender Faktoren) ein Vergleich der Rahmenbedingungen des Debt-Equity-Swap auf der Grundla- 
ge deutschen Rechts und auf der Grundlage englischen Rechts erforderlich. Auch in diesem Zusammenhang können die Untersuchungsergebnisse von Interesse sein.

\section{B. Zusammenfassung der Untersuchungsergebnisse}

Die Untersuchung hat ergeben, dass hinsichtlich einiger der untersuchten Problemkreise Debt-Equity-Swaps auf der Grundlage englischen Rechts unter erleichterten rechtlichen Rahmenbedingungen umsetzbar sind, als auf der Grundlage deutschen Rechts. Dies gilt entweder, weil spezifische Risiken bei Geltung deutschen Rechts nach englischem Recht nicht in entsprechender Weise bestehen, oder weil das englische Recht für Debt-EquitySwaps Gestaltungen ermöglicht, die das deutsche Recht nicht zulässt. Hervorzuheben sind diesbezüglich die fehlenden Einschränkungen aus einer der deutschen Lehre von der verdeckten Sacheinlage vergleichbaren Rechtsprechungstendenz oder gesetzlichen Regelung im englischen Recht (Kapitel IV), die Erleichterungen im Zusammenhang mit der Entbehrlichkeit der Beachtung strengerer Sachkapitalerhöhungsvorschriften bei der Anteilsausgabe zur Umsetzung des Debt-Equity-Swap nach englischem Recht (Kapitel IV), die (im Vergleich zu außergerichtlichen Debt-Equity-Swaps nach deutschem Recht) geringere Gefahr einer Differenzhaftung des forderungsumwandelnden Gläubiger infolge einer Überbewertung des Einlagewerts seiner Forderung nach englischem Recht (Kapitel IV), die Erleichterungen infolge der mangelnden Subordinierung von Gesellschafterdarlehen nach englischem Recht (Kapitel V) und die potentiellen Effizienzvorteile des Company Voluntary Arrangement (CVA) gemäß ss. 1 ff. IA 1986 und des Scheme of Arrangement gemäß ss. 895 ff. CA 2006 im Vergleich zum deutschrechtlichen Insolvenzplanverfahren mit Blick auf die Eignung dieser Verfahren als Instrument zur zwangsweisen Verpflichtung dissentierender Minderheitsgläubiger zur Forderungsumwandlung (Kapitel VII).

Die Untersuchung hat andererseits aber auch aufgezeigt, dass Debt-Equity-Swaps auf der Grundlage englischen Rechts nicht unter jedem untersuchten Aspekt unter erleichterten rechtlichen Rahmenbedingungen umsetzbar sind, als auf der Grundlage deutschen Rechts. Dies verdeutlichen insbesondere die Untersuchungsergebnisse zur übernahmerechtlichen Pflichtangebotsproblematik (Kapitel VI). Mit der übernahmerechtlichen Pflichtangebotsproblematik wurde eine rechtliche Hürde identifiziert, die infolge einer Teilharmonisierung der maßgeblichen Rechtsgrundlagen bei der Umsetzung von Debt-Equity-Swaps nach deutschem und nach englischem Recht gleichermaßen bestehen kann, aber auch gleichermaßen beherrschbar ist. Hinsichtlich der seit Inkrafttreten des ESUG bestehenden Effizienzvorteile des Insolvenzplanverfahrens im Vergleich zum Company Voluntary Arrangement und zum Scheme of Arrangement als verfahrensrechtliches Instrument zur Reduktion des Blockadepotentials der Altgesellschafter wurden jedoch auch Erleichterungen beim Debt-Equity-Swap nach deutschem Recht festgestellt, die beim Debt-Equity-Swap nach englischem Recht nicht bestehen (Kapitel VIII, sowie sogleich).

Vor diesem Hintergrund lässt sich bei einer Gesamtwürdigung der Untersuchungsergebnisse nicht pauschal resümieren, dass Debt-Equity-Swaps zur Entschuldung sanierungsbedürftiger Kapitalgesellschaften auf der Grundlage englischen Rechts unter erleichterten rechtlichen Rahmenbedingungen umsetzbar sind, als auf der Grundlage deutschen Rechts.

Trotz ebenfalls identifizierter Regelungsdefizite des ESUG bewirken die untersuchten Neuerungen eine Erleichterung der deutschrechtlichen Rahmenbedingungen hinsichtlich der Umsetzung von Debt-Equity-Swaps zur Entschuldung krisenbefangener Kapitalgesellschaften und führen insoweit zu einer erheblichen Steigerung der Rechts- und Transakti- 
onssicherheit für die Restrukturierungspraxis. Mit Blick auf die (vermeintlichen oder tatsächlichen) Wettbewerbsnachteile des deutschen Insolvenz- und Sanierungsrechts in Gestalt von unvorteilhafterer Rahmenbedingungen für Debt-Equity-Swaps auf der Grundlage deutschen Rechts, im Vergleich zu solchen auf der Grundlage englischen Rechts, wurden durch das ESUG punktuell Vorteile des englischen Rechts nivelliert (etwa bei der Differenzhaftung für insolvenzplangestützte Debt-Equity-Swaps) oder sogar deutschrechtliche Hürden abgebaut, die nach englischem Recht fortbestehen. Letzteres gilt insbesondere hinsichtlich der Eignung der verfahrensrechtlichen Instrumente zur effizienten Reduktion des Blockadepotentials obstruierender Anteilseigner im Interesse der Erleichterung von Debt-Equity-Swaps.

Die Arbeit enthält jeweils am Ende ihrer Kernkapitel (Kapitel IV-VIII) eine Zusammenfassung der wesentlichen Untersuchungsergebnisse und Stellungnahmen. Die wichtigsten Ergebnisse lassen sich thesenartig wie folgt zusammenfassen:

1. Der Hauptunterschied der für Debt-Equity-Swaps relevanten Kapitalaufbringungsvorschriften deutschen und englischen Rechts besteht darin, dass die Forderungseinbringung im Recht der plc und Ltd. als Bareinlage, im deutschem Aktien- und GmbH-Recht dagegen nach ganz h.M. als Sacheinlage behandelt wird (dazu auch nachfolgend 2. und 3.).

2. Nach deutschem Aktien- und GmbH-Recht ist die Einbringung einer gegen die kapitalerhöhende Gesellschaft gerichteten Forderung als Sacheinlage zulässig, aber infolge extensiv ausgelegter Kapitalaufbringungsregeln auch erforderlich. Eine Gegenansicht, wonach das MoMiG den Weg für eine von den bisherigen Grundsätzen abweichende Behandlung der Kapitalaufbringung beim Debt-Equity-Swap eröffnet habe und nunmehr auch die Mittel einer Barkapitalerhöhung generell zur Bezahlung von Altschulden der Gesellschaft beim Inferenten verwendet werden dürften (ohne als verdeckte Sacheinlagen sanktioniert zu werden) ist erheblichen Bedenken ausgesetzt und daher abzulehnen.

3. Auf der Grundlage des englischen Rechts der plc und Ltd. wird die Forderungseinbringung bei einem Debt-Equity-Swap im Wege einer Kapitalerhöhung als zulässige Tilgungsmodalität der Bareinlageforderung qualifiziert. Hierin wird nach englischem Recht keine unzulässige Umgehung der Sachkapitalerhöhungsvorschriften gesehen, über der das Damoklesschwert einer, der deutschen Lehre von der verdeckten Sacheinlage vergleichbaren Rechtsprechungstendenz oder gesetzlichen Regelung schweben würde.

4. Im Vergleich zum deutschen Kapitalgesellschaftsrecht mit dem Erfordernis der Einhaltung strengerer Sachkapitalerhöhungsvorschriften stellt das englische Kapitalgesellschaftsrecht mit der Entbehrlichkeit der Werthaltigkeitsprüfung des Einlagewerts der einzubringenden Forderung und dem damit verbundenen geringeren Zeit- und Kostenaufwand (unter diesem Gesichtspunkt) erleichterte rechtliche Rahmenbedingungen für Debt-Equity-Swaps zur Verfügung.

5. Hinsichtlich der Forderungseinbringung bei einem Debt-Equity-Swap nach Maßgabe des englischen Kapitalgesellschaftsrechts ist davon auszugehen, dass bei der Prüfung einer etwaigen Unterpari-Emission auch dann (noch) zulässigerweise auf den Nominalwert der nach sec. 583(3)(c) CA 2006 einzubringenden Forderung abzustel- 
len ist, wenn der Verkehrswert dieser Forderung aufgrund der Liquiditätskrise der Schuldnergesellschaft im Zeitpunkt des Debt-Equity-Swap geringer ist als ihr Nominalwert. Im Vergleich hierzu erschwert es Debt-Equity-Swaps nach deutschem Kapitalgesellschaftsrecht, dass hier de lege lata beim (außerinsolvenzlichen und insolvenzplangestützten) Debt-Equity Swap nicht von der Zulässigkeit der generellen Nennwertanrechnung von Forderungen nach Maßgabe der Nennwertthese auszugehen ist, sondern bei der Forderungsbewertung der Verkehrswert der einzulegenden Forderung maßgeblich ist.

6. Durch die Einführung der Befreiung von der Differenzhaftung nach $\S 254$ Abs. 4 InsO ist es dem ESUG partiell gelungen, als Wettbewerbsnachteile geltenden Defizite des deutschen Insolvenzrechts im Vergleich zum englischen Recht abzumildern, weil sich nach deutschem Recht - wie bei Debt-Equity-Swaps nach englischem Recht - nunmehr zumindest für insolvenzplangestützte Debt-Equity-Swaps die Gefahr einer Differenzhaftung des forderungsumwandelnden Gläubigers ausschließen und insoweit Transaktionssicherheit erzielen lässt. Weil die Gefahr einer Differenzhaftung bei Debt-Equity-Swaps nach deutschem Recht jedoch (anders als nach englischem Recht) nur innerhalb des Insolvenzplanverfahrens einfach ausgeschlossen werden kann, stellt das englische Recht bei einer Fokussierung des Blicks auf diesen Aspekt auch nach Inkrafttreten des ESUG die vorteilhafteren rechtlichen Rahmenbedingungen für Debt-Equity-Swaps zur Verfügung.

7. Das Einrücken in die Gesellschafterposition infolge eines Debt-Equity-Swap birgt nach englischem Recht keine Gefahr, dass nicht umgewandelte Darlehensforderungen oder Forderungen aus neu gewährten Darlehen bei Scheitern der Sanierungsbemühungen in einem späteren Insolvenzverfahren generell als nachrangige Forderungen behandelt werden. Bei der Umsetzung von Debt-Equity-Swaps zur Entschuldung einer $\mathrm{AG}$ oder $\mathrm{GmbH}$ stehen den hieraus resultierenden Vorzügen des englischen Rechts diejenigen Nachteile für forderungsumwandelnde Gläubiger gegenüber, die ihnen nach deutschem Recht aus den Nachrangigkeits- und Anfechtungsregeln für Gesellschafterdarlehen ( $\$ \S 39$ Abs. 1 Nr. 5 und 135 InsO) drohen. Die deutschen Ausnahmetatbestände des Kleinbeteiligungsprivilegs und des Sanierungsprivilegs verringern diese Nachteile zwar, dies jedoch mit spezifischen Einschränkungen.

8. Aus 7. folgt: Mit Blick auf den Aspekt des rechtlichen Schicksals von Gesellschafterdarlehen im Fall der Insolvenz der Gesellschaft wird es für Gläubiger/Investoren attraktiver sein, ihre gegen die Gesellschaft gerichteten Forderungen im Rahmen eines Debt-Equity-Swap auf der Grundlage englischen Kapitalgesellschafts- und Insolvenzrechts in Anteile an der Schuldnergesellschaft umzuwandeln, als sich an einem Debt-Equity-Swap auf der Grundlage deutschen Rechts zu beteiligen. Denjenigen Gläubigern/Investoren, für die die Vermeidung des Risikos einer faktischen Entwertung nicht umgewandelter Darlehensforderungen von zentraler Bedeutung ist, könnten die begrenzten Handlungsspielräume, die das deutsche Sonderrecht für Gesellschafterdarlehen bei einem Anteilserwerb im Zuge des Debt-Equity-Swap eröffnet, zu eng erscheinen. Unter diesem Gesichtspunkt erschwert das deutsche Recht die Umsetzung von Debt-Equity-Swaps im Vergleich zum englischen Recht.

9. Im Kontext der Umsetzung von Debt-Equity-Swaps bei Gesellschaften im Anwendungsbereich des deutschen WpÜG und im Anwendungsbereich des englischen City Code, können sich die - konzeptionell sehr ähnlichen - Pflichtangebotsregelun- 
gen in $\S 35$ Abs. 2 S. 1 WpÜG und Rule 9 City Code gleichermaßen nachteilig auf die Erfolgsaussichten des Debt-Equity-Swap auswirken.

10. Die seit Inkrafttreten des ESUG von einigen Autoren vertretene Ansicht, dass im Fall der Umsetzung eines Debt-Equity-Swap einer börsennotierten AG auf der verfahrensrechtlichen Grundlage eines Insolvenzplanverfahrens die Bestimmungen des WpÜG zur Angebotspflicht entbehrlich seien, da die Angebotspflicht nach Maßgabe des WpÜG dem Schutz der Kleinaktionäre diene und dieser im Insolvenzplanverfahren über $§ 251 \mathrm{InsO}$ gewährleistet sei, ist abzulehnen.

11. Die praktische Anwendung der Zurechnungstatbestände des abgestimmten Verhaltens gemäß $\S 30$ Abs. 2 S. 1 WpÜG und des acting in concert i.S.d. Rule 9.1 City Code ist im Kontext der Umsetzung eines Debt-Equity-Swap mit einem ähnlich hohen Maß an Rechtsunsicherheit für die Beteiligten verbunden.

12. Die Erzielung der Befreiung forderungsumwandelnder Gläubiger von der Angebotspflicht im Anwendungsbereich des WpÜG und des City Code ist regelmäßig von der Erfüllung der gänzlich unterschiedlichen Voraussetzungen der Sanierungsbefreiung nach WpÜG/WpÜG-AngVO und der whitewash procedure des City Code abhängig. Dies unter anderem, weil im Anwendungsbereich des City Code der SanierungsBefreiungstatbestand der rescue operations gemäß Note 3 der Dispensation Notes zu Rule 9 City Code bei Debt-Equity-Swaps zu Sanierungszwecken lediglich subsidiär zur whitewash procedure Anwendung findet.

13. Weil die Voraussetzungen der Schlüssel-Befreiungstatbestände des WpÜG/WpÜGAngVO und des City Code zwar unterschiedlich, aber im Grundsatz erfüllbar sind, ist der Erwerb einer Kontrollbeteiligung des forderungsumwandelnden Gläubigers im Zuge eines Debt-Equity-Swap i.d.R. im Anwendungsbereich beider Pflichtangebotsregelungen auch ohne das Auslösen einer Angebotspflicht möglich. Hinsichtlich der bestehenden rechtlichen Hürden der Umsetzung von Debt-Equity-Swaps bei Kapitalgesellschaften im Anwendungsbereich des WpÜG einerseits und bei Kapitalgesellschaften im Anwendungsbereich des City Code andererseits ist jedenfalls unter dem Aspekt der Pflichtangebotsproblematik kein wesentliches Gefälle festzustellen.

14. Das Insolvenzplanverfahren gemäß $\S 217$ ff. InsO, das Company Voluntary Arrangement (CVA) gemäß ss. 1 ff. IA 1986 und das Scheme of Arrangement gemäß ss. 895 ff. CA 2006 enthalten zwar allesamt Mechanismen zur Linderung von Kollektivhandlungsproblemen auf Gläubigerseite, jedoch sind diese Verfahren als Mittel zur notfalls zwangsweisen Verpflichtung einzelner dissentierender Gläubiger zur Forderungsumwandlung unterschiedlich effizient (dazu nachfolgend 15.-19.).

15. Das $C V A$ ist im Allgemeinen, d.h. ohne Berücksichtigung der Sonderkonstellation des Debt-Equity-Swaps, ein weniger effizientes Instrument zur zwangsweisen rechtlichen Bindung dissentierender Minderheitsgläubiger an einen Sanierungsplan, als das deutsche Insolvenzplanverfahren. Denn - anders als das Insolvenzplanverfahren - ermöglicht das CVA keine zwangsweise rechtliche Bindung (cram-down) einer ganzen, den Sanierungsplan im Abstimmungsverfahren mehrheitlich ablehnenden Gläubigergruppe und von Gläubigern mit gesicherten Forderungen. 
16. Die Effizienzvorteile des deutschen Insolvenzplanverfahrens im Vergleich zum CVA mit Blick auf die Eignung beider Verfahren zur Überwindung der Obstruktion dissentierender Minderheitsgläubiger sind in der Sonderkonstellation, in der die zwangsweise Verpflichtung einzelner Gläubiger zur Umwandlung ihrer gegen die Gesellschaft gerichteten Forderungen in Gesellschaftsanteile beabsichtigt ist, nicht nutzbar. Grund hierfür sind die Auswirkungen der individuellen Zustimmungserfordernisse bei Debt-Equity-Swaps nach $\S 225$ a Abs. 2 S. 2 InsO und (wie bereits vor Inkrafttreten des ESUG) nach $\S 230$ Abs. 2 InsO, denen im CVA-Verfahren kein Äquivalent gegenübersteht. Im Vergleich zum deutschen Insolvenzplanverfahren eröffnet das CVA insoweit größere Handlungs- und Gestaltungsspielräume zur Erzwingung einer Forderungsumwandlung von einzelnen dissentierenden Minderheitsgläubigern beim Debt-Equity-Swap.

17. Wird ein Debt-Equity-Swap auf der Grundlage eines Scheme of Arrangement gemäß ss. 895 ff. CA 2006 umgesetzt, so können sich im Vergleich zur Umsetzung im deutschen Insolvenzplanverfahren die bereits zum CVA-Verfahren skizzierten Vorteile aus der Nicht-Existenz einer den $\S \S 225 a$ Abs. 2 S. 2, 230 Abs. 2 InsO im Regelungsgehalt entsprechenden Vorschrift oder Rechtsprechungsregel ergeben.

18. Das cram-down-Potential des Scheme of Arrangement ist auf die Majorisierung Einzelner durch mehrheitliche Zustimmung in den einzelnen Gläubigergruppen beschränkt; der cram-down einer ganzen Abstimmungsgruppe von Gläubigern, die dem Scheme of Arrangement nicht mit der erforderlichen Mehrheit zustimmt, ist (wie im $C V A$-Verfahren) nicht möglich, so dass einer ganzen Abstimmungsgruppe eine Beteiligung an einem Debt-Equity-Swap nicht aufgezwungen werden kann. Etwas anderes folgt nicht aus der Rechtsprechung englischer Gerichte zur ,economic interest issue “.

19. Beim Einsatz als verfahrensrechtliche Grundlage eines Debt-Equity-Swap sind CVA und Scheme of Arrangement - auch nach Inkrafttreten des ESUG - insoweit besser als das deutsche Insolvenzplanverfahren zur Bewältigung von Kollektivhandlungsproblemen auf der Gläubigerseite und gleichzeitiger Vermeidung einer Überschreitung des Zeitpunkts der Sanierbarkeit geeignet, als sie die Bewältigung von Kollektivhandlungsproblemen auf der Gläubigerseite in einem früheren Krisenstadium ermöglichen. Etwas anderes folgt nicht aus der Einführung des „Schutzschirmverfahrens" gemäß $§ 270 b$ InsO durch das ESUG, denn das Schutzschirmverfahren als lediglich „sanierungsvorbereitendes Eröffnungsverfahren“ ermöglicht keine Eingriffe in Gläubigerrechte (und somit auch keinen cram-down) im Zeitraum vor Eröffnung des Insolvenzverfahrens.

20. Das $C V A$ ist zur effizienten Reduktion des Blockadepotentials der Altgesellschafter bei der Umsetzung eines Debt-Equity-Swap im Zuge einer Reorganisation der Schuldnergesellschaft nicht geeignet und insofern ineffizienter als das Insolvenzplanverfahren gemäß $\S 217 \mathrm{ff}$. InsO in der durch das ESUG reformierten Fassung.

21. Das Scheme of Arrangement ist unter dem Gesichtspunkt des Blockadepotentials der Altgesellschafter bei der Umsetzung eines Debt-Equity-Swap im Vergleich zum Insolvenzplanverfahren i.d.F. des ESUG ineffizienter, weil es - anders als das Insolvenzplanverfahren - keine zwangsweise rechtliche Bindung (cram-down) einer gan- 
zen Abstimmungsgruppe von Gesellschaftern, die dem Scheme of Arrangement nicht mit erforderlicher Mehrheit zustimmt, ermöglicht.

22. Hinsichtlich der Effizienz der verfahrensrechtlichen Instrumente zur Reduktion des Blockadepotentials der Altgesellschafter bei der Umsetzung von Debt-Equity-Swaps (oder anderer Sanierungsmaßnahmen) ist seit Inkrafttreten des ESUG nicht nur kein Standortnachteil des deutschen Rechts im Vergleich zum englischen Recht mehr feststellbar, sondern vielmehr umgekehrt ein Standortnachteil des englischen Rechts im Vergleich zum deutschen Recht. Dies ist als Verdienst des ESUG zu werten, das insoweit Effizienzdefizite des deutschen Insolvenzplanverfahrens erfolgreich abgebaut hat, während entsprechende Reformvorschläge für das englische Recht bisher erfolglos blieben. 